\title{
Developed third iterative dichotomizer based on feature decisive values for educational data mining
}

\author{
Saja Taha Ahmed ${ }^{1}$, Rafah Al-Hamdani ${ }^{2}$, Muayad Sadik Croock ${ }^{3}$ \\ ${ }^{1,2}$ The Informatics Institute for Postgraduate Studies, Iraqi Commission for Computers \& Informatics (IIPS-ICCI) \\ ${ }^{3}$ Computer Engineering Department, University of Technology, Iraq
}

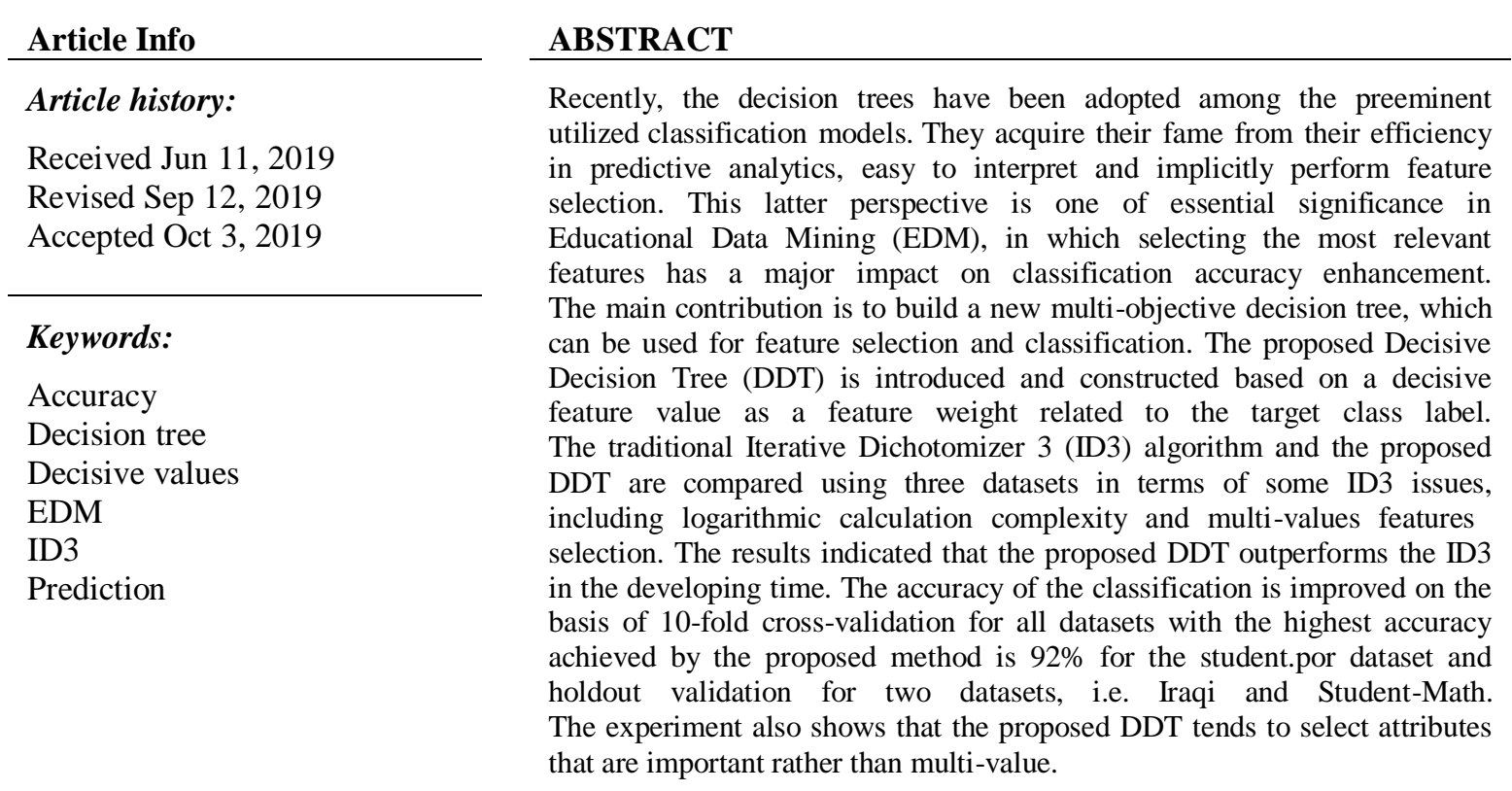

Copyright $(2020$ Institute of Advanced Engineering and Science. All rights reserved.

\section{Corresponding Author:}

Saja Taha Ahmed,

The Informatics Institute for Postgraduate Studies,

Iraqi Commission for Computers \& Informatics, Baghdad, Iraq.

Email: sajataha@ymail.com ; sajataha2@yahoo.com

\section{INTRODUCTION}

Educational Data Mining (EDM) is employed to extract the relevant information from the extensive and complex educational datasets and it is valuable for data analysis and predictions [1]. The prediction is commonly applied using EDM that considers the following techniques: classification, clustering, association rule mining, etc. Classification is the most popular EDM methodology used for student performance prediction. There are numerous classification methods that can be categorized such as decision tree, neural network, $\mathrm{k}$ Nearest neighbor, etc. These techniques are typically accustomed to building the classification model, which predicts the future trend based on the previous pattern [2-3].

The decision tree is a foremost widespread methodology for data classification, which incorporates numerous types, such as Third Iterative Dichotomizer (ID3) that selected optimal attribute using information gain [4]. Different decision tree methods are developed from the ID3 method, such as C4.5 based on gain ratio [5], as well as Classification and Regression Tree (CART) used Gini index [6].

In general, the decision tree assists educational institutions and universities in decision making in order to provide a student with the necessary assistance in the learning process. It is so popular because complex data can be presented in a visual representation with all possible outcomes and produce 
classification rules that are easy to interpret than other classification methods. The most relevant subset features for a decision automatically emerge through the process of developing the tree, the top nodes of the tree are the most essential, since they are deciding the subsequent decisions to be made. In addition, the tree demonstrates the order decisions must be made and eliminates ambiguity related to how each item influences the others [7]. Nevertheless, ID3 specifically, has some burdens, such as:

a) It is time-consuming due to information entropy calculation which is based on logarithmic algorithms [8-9] since the computation speed of the logarithmic expression is slower than four arithmetic operations that only include adding, subtract, multiply and divide [10].

b) It uses information gain as attribute selection criteria that pick the multi-values attribute, and the number of attribute values cannot be used to measure the attribute significance. This major shortcoming influences the accuracy of the decision tree [11].

c) The Decision tree can have overfitting, a phenomenon in which a model becomes more complex. When it is excessively dependent on irrelevant attributes of the training data, the result is that it works well on the training data but is relatively poorly predictive on unseen instances [12].

Over the past few years, a number of researchers have presented many related works for the use and/or suggestion of an enhancement in decision tree methods of various classification problems, below are some of the related works in this field.

ID3 has some exist disadvantages such as tending to select attributes biasing towards multi-values. The logarithmic expression has a high complexity computation and large-scale size. The authors of [13] proposed an improved ID3 algorithm that combines the simplified information entropy based on different weights with coordination degree in rough set theory. The traditional ID3 and the improved one are compared by exploiting three datasets, the experimental results showed that the proposed algorithm outperformed in the running time and tree size, but not in classification accuracy for small datasets.

The ID3 uses information gain tend to select the attribute with more values but it cannot measure the attribute importance via the number of attribute values. Therefore, the authors of [14] proposed a new method that selected the splitting attribute based on the utilization of conditional probability calculation of close contact between the attributes and the decision attributes. It joined with information gain to get higher predictive accuracy and less number of leaves without taking into consideration the running time. In perspective of the above issue, the authors of [15] suggested normalized association function combined with gain for each attribute to decide splitting decision, this can enhance accuracy but increase time complexity for proposed decision tree.

This paper aims to create a classification model particularly a decision tree algorithm that can effectively characterize students into one of two classes (Pass or Fail) by predicting the future grades of the students in their final examinations. The proposed algorithm aims to identify significant factors influencing student achievement and addresses the mentioned ID3 problems. A new methodology is utilized to build the proposed Decisive Decision Tree (DDT) based on the fact that the evaluation must consider the combination between the relevancy degree of each feature and the degree of classification accuracy enforcement. Therefore, the features relevancy degrees and the existing cross coupling are evaluated when they are combined together based on feature decisive (weighting) values. The proposed mechanism is examined by three datasets, namely, Iraqi dataset and UCI student performance dataset that includes mathematics, and Portuguese language courses datasets. The experimental results show that the proposed DDT obtains better performance than traditional ID3, in terms of, classification accuracy, running time and optimum multi-value feature selection.

\section{RESEARCH METHOD}

This study will include two phases as a part of methodology, as follow:

\subsection{Dataset Collection}

As mention earlier, this study incorporates three datasets. The first dataset is called Iraqi dataset which is uploaded at [16] and used for EDM preprocessing and Neural Network classification by [17]. It is collected during the second semester of 2018 by applying (or submitting) questionnaire in three Iraqi secondary schools for the applicable and biological branches of the final stage. The questionnaire initially contains 56 questions in three A4 sheets and 250 students (samples) respond to the questionnaire. Later, 130 samples are discarded due to lack of information, as pre-processing is used to obtain students ' most complete information. This study considers 120 instances with 55 features for experimental purposes after removing inconsistencies and incompleteness in the dataset. The attributes are divided into five main categories: Demographic, Economic, Education, Time and Marks. Furthermore, new features such as holidays and 
worrying effects are introduced. Also, the relationships between parents and schools and the student's use of books and references are considered.

The second used dataset in this study is (Student Alcohol Consumption Data Set), obtained from UCI Portugal [17-18]. This data set was collected during the 2005-2006 year from two public schools depending on two sources: school reports for the three-period grades and number of school absences, and questionnaires. The dataset consists of two datasets: student-mat.csv (Math), which holds 395 instances of Math course) and student-por.csv (Por), which holds 659 instances of Portuguese language course. Both of these datasets, consisting of 32 attributes.

\subsection{The Proposed Methodology}

A new criterion to build a decision tree for student performance prediction is presented. The Decisive Feature (Weight) value was calculated for both the training and the test set depending on the relative probability of the existing features occurring with respect to the target class.

The first stage is DDT building, in which the proposed system introduces the idea of obtaining each attribute in training set an importance via testing its significant degree with target class using the feature weight value calculated for each of the attributes, initially (1) [19-20] is used to compute a significant degree for target class:

$$
D_{t}=\frac{\text { Ftsuccess }- \text { Ftfail }}{\text { Ftsuccess }+ \text { Ftfail }}
$$

Where; $\mathbf{t}$ is a target class.

$\mathbf{D}_{\mathbf{t}}$ is the Decisive value of the target.

Ftsuccess is the frequency of occurrence of success class.

Ftfail is the frequency of occurrence of fail class.

The decisive values of the attributes are considered as leading indicators for feature weighting and significance analysis for the student's success/failure prediction task. The Decisive value (D) is within [ 1, -1] range. If the value is approximately 1 , it implies that most of the feature is done with a successful student class. If the value is approximately to -1 , it implies that the feature generally happens with a failure student class. While the value is near to 0 , it implies that the feature in the success class is almost equivalent to failure class.

The Cumulative Decisive value (CD) is computed using (2) by multiplying the D value of each attribute's category with its frequency. This takes into account the volume of the frequent occurrence of values that construct a specific attribute in relation to the target class.

$$
\mathrm{CD}(\mathrm{i})=\sum_{j=1}^{N}\left(\mathrm{D}(\mathrm{ij}) * \frac{\text { Frequancy of Occurance of Value } j}{\text { Total Number of Values within Attribute } i}\right)
$$

Where; $\mathbf{i}$ is a specific attribute.

$\mathbf{j}$ is a value within attribute $\mathbf{i}$.

$\mathbf{N}$ is the number of values (categories) within attribute $\mathbf{i}$.

$\mathrm{D}(\mathrm{ij})$ is the Decisive value of specific category $\mathrm{j}$ within attribute $\mathrm{i}$, the (1) of the target becomes (3) for attribute categories, with the description of the following parameters:

$$
D(i j)=\frac{\text { Fisuccess }(\mathrm{ij})-\text { Fifail }(\mathrm{ij})}{\text { Fisuccess }(\mathrm{ij})+\text { Fifail }(\mathrm{ij})}
$$

Fisuccess (ij) is the frequency of occurrence of value $\mathrm{j}$ of attribute $\mathrm{i}$ in success class. Fifail (ij) is the frequency of occurrence of value $\mathrm{j}$ of attribute $\mathrm{i}$ in a fail class.

Finally, the best attribute is selected using Gain by subtracting CD for each attribute from the target Dt using (4). The highest attribute gain is recommended to be the best attribute placed at the root for further splitting. The proposed DDT is continued in this way by testing every property with others until pure target class (all success or failure) is reached or no further splitting is found. In the latter case, when there is no combination of the values of attributes along the current path. The proposed DDT takes into consideration $\mathrm{D}$ (ij) for a specific category (current value) in the original training set, which has no combination within this path. Then DDT decides whether the leaf node will succeed or fail, if D(ij) value predominantly closes 1 , at that point, the decision will succeed, otherwise, the decision will fail, this has a major impact on the tree classification accuracy enhancement. In contrast to traditional DT, which depends on the majority of the target class label when there is no combination of values (i.e. samples(value) is empty) and ignores the 
weight of current category on the classification. The important steps for building the proposed DDT, are illustrated in Algorithm (1).

$$
\operatorname{Gain}(i)=D_{t}-C D(i)
$$

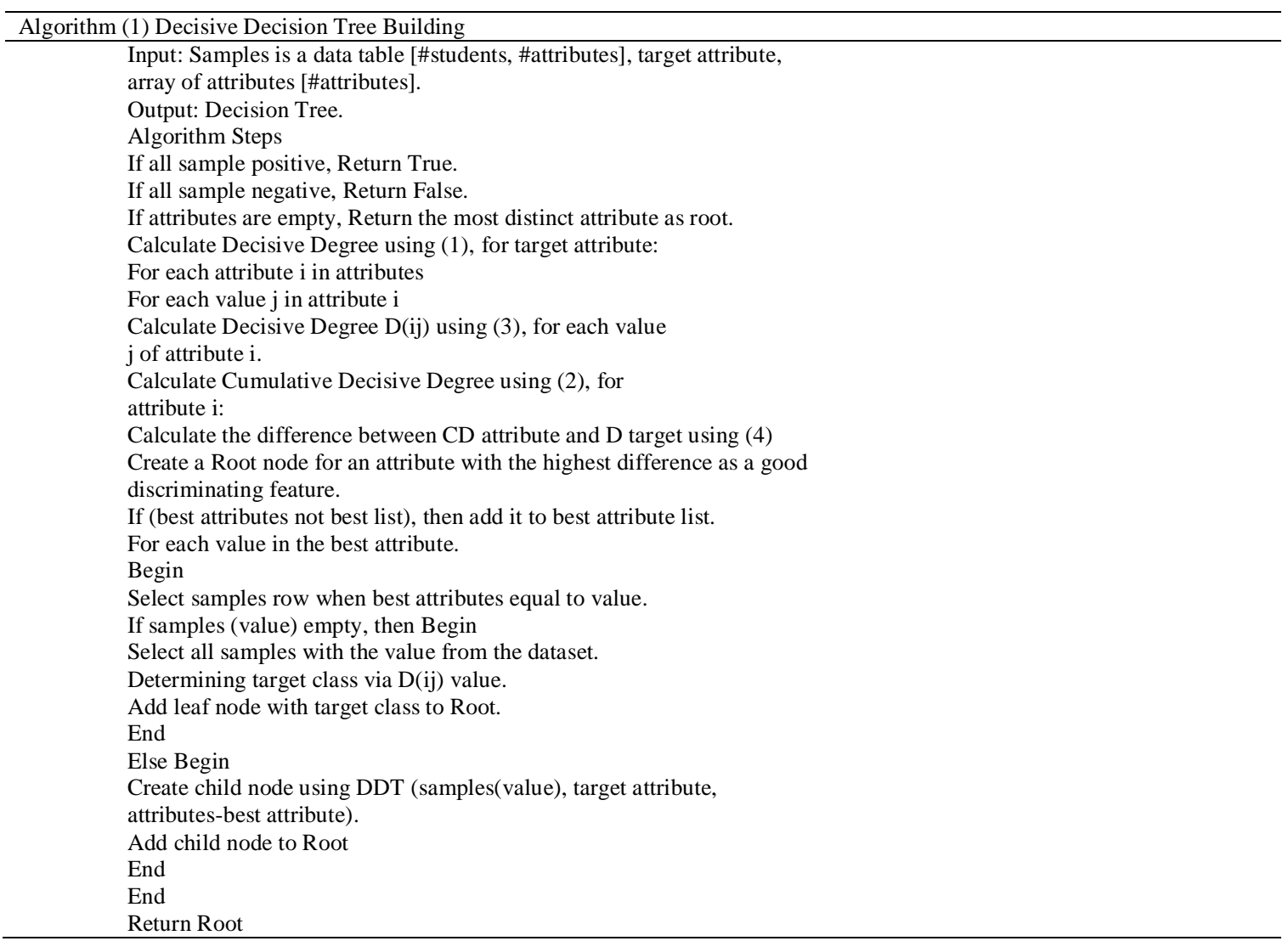

In the second stage, when a DDT is generated, the target class prediction for a new student in the test set is determined and the classification rules can be extracted using the DDT search clarified in the algorithm (2). Each new student information enters as a matrix of two tuples, tuple 0 contains the name of the attributes, and tuple 1 contains values corresponding to the attributes. DDT search mainly depends on matching student information at each node and tracing the path from the root to the target class at a leaf node.

Algorithm (2) DDT Search

Input: Root, new student information as string test [2, \#attributes] $] /$ row

0 : name of an attribute, row 1 values of each attribute

Output: Path for a new student in the test set.

Algorithm Steps

Step1: Define index as -1 and tag as False.

For each attribute $i$ in the test set

If test $[0, i]$ equal to Root. Attribute

Begin

Set index to i; Break.

End

Set Path to Root.Attribute + test [1, index]

If Root.Attribute. Values not equal to Null

Begin

For each value $\mathrm{j}$ in attribute

If test [1, index] equal to Root.Attribute. Values[j]

Begin

Set Val to $\mathrm{j}$

Set Tag to True; Break;

End

If Tag equal to True

Begin 


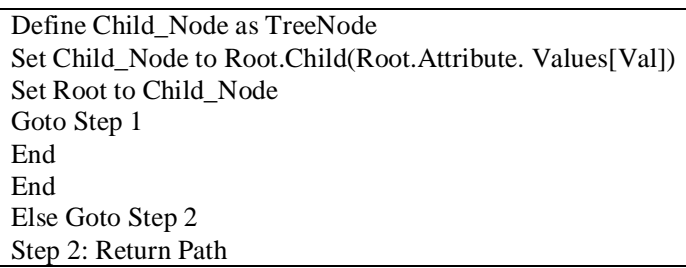

\section{RESULTS AND ANALYSIS}

The experiments and the application system in this study are developed based on visual studio C\# 2015. The model validation empowers locating the best features of the model while also shielding it from getting the chance to be over fitted. The proposed DDT model is assessed utilizing two of the most popular evaluation criteria 10-fold cross-validation and hold out methods. In 10-fold cross-validation [21], all the dataset has been divided into 10 subsets of approximately equal size. This is an iterative procedure, each time 9 subsets acts as a training data and one set is used as a testing data. In the holdout method [22], the data set is separated into two sets of training data is $70 \%$ of the entire dataset and testing data is $30 \%$, represents the remaining dataset.

Since the decision tree needs the data to be in the categorical formulation, the grade features must have discrete values to obtain better results. The discretization mechanism has been exploited to convert the grade values from numerical values to nominal ones. Specific classes are defined, which represent classes label for student performance prediction, which can be either "Pass" or "Fail". In UCI dataset, there are three average G1, G2 and G3 have ranged from 0 to 20. Thus, if the student has average equal or higher than 10, it should be defined within the "Pass" label, otherwise should be defined as "Fail" student. In Iraqi dataset, grade scores are within range $0-100$, if the student has average equal or higher than 50 , it should be defined within "Pass" label, otherwise is classified as "Fail" student.

A small training data set is examined to illustrate the difference between the structure of ID3 and DDT algorithms. Table 1 shows the dataset used in research work [14].

Table 1. The Dataset

\begin{tabular}{|c|c|c|c|c|c|c|}
\hline ID & Chinese & Mathematics & English & Physics & Summary & Target Class \\
\hline 1 & general & good & bad & general & qualified & $\mathrm{Q}$ \\
\hline 2 & general & good & good & good & qualified & Q \\
\hline 3 & good & general & general & good & qualified & $\mathrm{Q}$ \\
\hline 4 & optimal & general & good & good & qualified & $\mathrm{Q}$ \\
\hline 5 & general & general & general & general & qualified & Q \\
\hline 6 & good & bad & general & bad & unqualified & $\mathrm{U}$ \\
\hline 7 & optimal & bad & bad & general & unqualified & $\mathrm{U}$ \\
\hline 8 & good & optimal & optimal & optimal & qualified & Q \\
\hline 9 & general & general & optimal & good & qualified & Q \\
\hline 10 & optimal & bad & general & general & qualified & Q \\
\hline 11 & bad & good & good & bad & unqualified & $\mathrm{U}$ \\
\hline 12 & good & general & good & good & qualified & $\mathrm{Q}$ \\
\hline 13 & general & bad & good & general & qualified & Q \\
\hline 14 & general & general & optimal & good & qualified & Q \\
\hline 15 & good & bad & good & general & qualified & Q \\
\hline 16 & optimal & general & optimal & good & qualified & Q \\
\hline 17 & optimal & optimal & optimal & optimal & qualified & $\mathrm{Q}$ \\
\hline 18 & good & bad & good & general & qualified & Q \\
\hline 19 & good & general & bad & optimal & qualified & Q \\
\hline 20 & general & general & general & general & qualified & $\mathrm{Q}$ \\
\hline
\end{tabular}

ID3 favors the selection of attribute that has a larger number of values (i.e. categories) because the attribute with more values has high information gain than others. Figure 1 shows the ID3 feature selection, which chooses the ID feature with 20 values as the root node for the decision tree.

The proposed DDT selects English attribute with four categories (bad, general, good, optimal) to be the root node of the decision tree and exclude ID as it has no predictive power of classification which explained in Figure 2. Since the proposed DDT tends to select the attribute that has high weight value regarding target labels, in the case of Table. 1 there are two target labels qualified and unqualified. 


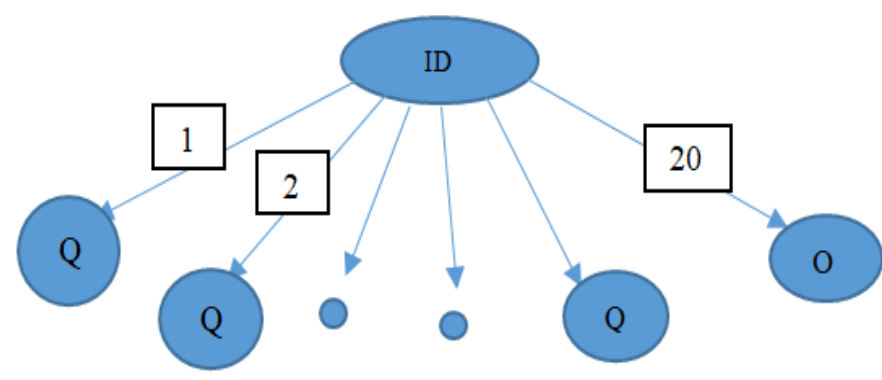

Figure 1. ID3 Decision tree construction

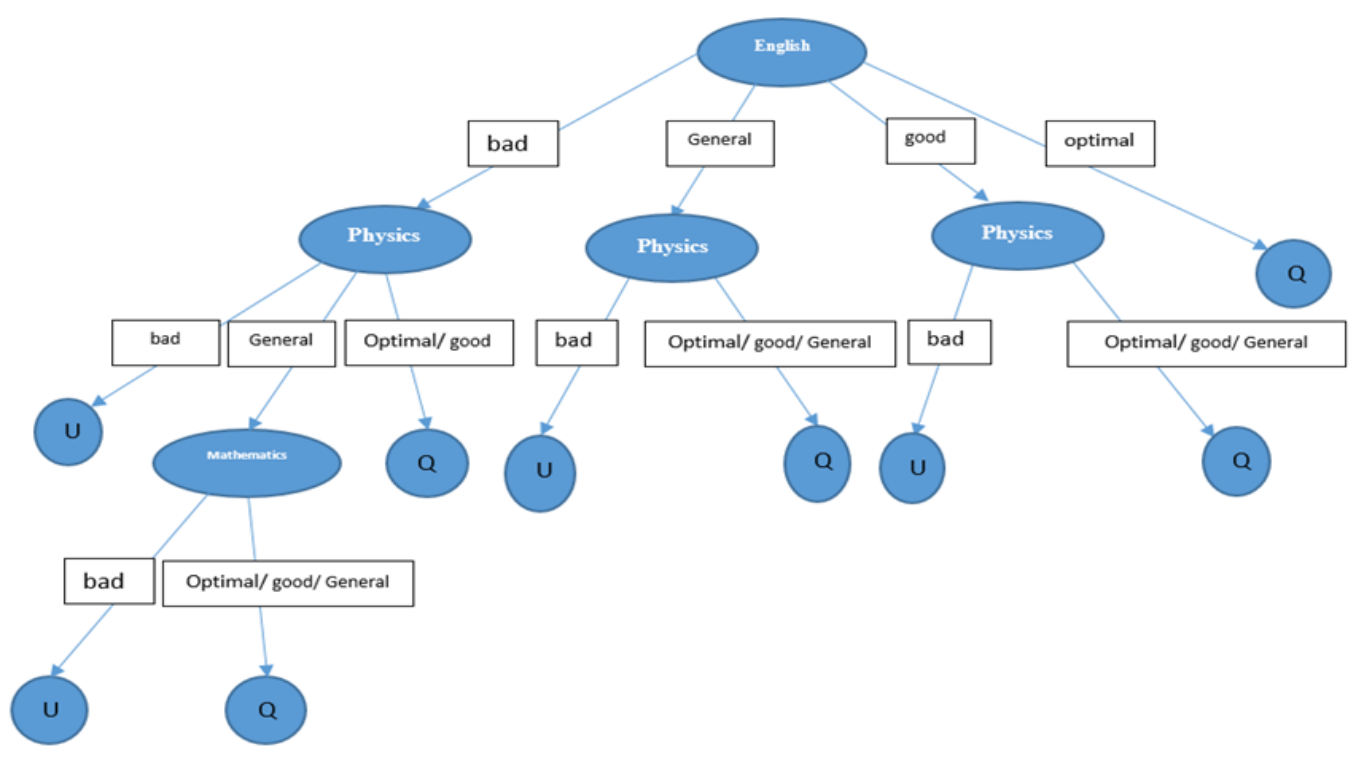

Figure 2. DDT Decision tree construction

The evaluation on the basis of Accuracy (ACC) value is executed. Accuracy measures the degree to which the instances correctly classified by machine learning algorithm and can be computed using a confusion matrix with (5) as follows [23]:

$$
A C C=\frac{\sum \text { True Positive }+\sum \text { True Negative }}{\sum \text { Total Population }}
$$

Holdout cross-validation for three datasets Iraqi dataset, Por, and Math depend on confusion matrix that can be illustrated in Tables of 2, 3, and 4 . It can be shown that the achieved accuracies of the predicted classes are $88.88,61.5$, and 74.7 , respectively.

Table 2. Confusion Matrix of Iraqi Dataset

\begin{tabular}{cccc}
\multicolumn{2}{c}{ Total Population $=36$} & \multicolumn{2}{c}{ Actual Calss } \\
\cline { 2 - 4 } Acc $=88.88$ & SUCCESS & FAIL \\
Prediction & SUCCESS & TP $=32$ & FP $=4$ \\
Class & FAIL & FN $=0$ & TN $=0$ \\
\hline
\end{tabular}

Table 3. Confusion Matrix of Por Dataset

\begin{tabular}{cccc}
\hline \multicolumn{2}{c}{ Total Population=195 } & \multicolumn{2}{c}{ Actual Calss } \\
\cline { 3 - 4 } Acc $=61.5$ & & SUCCESS & FAIL \\
\hline Prediction & SUCCESS & TP $=105$ & FP $=70$ \\
Class & FAIL & FN $=5$ & TN $=15$ \\
\hline
\end{tabular}

Table 4. Confusion Matrix of Math Dataset

\begin{tabular}{cccc}
\hline \multicolumn{2}{c}{ Total Population $=119$} & \multicolumn{2}{c}{ Actual Calss } \\
\cline { 3 - 4 } Acc $=74.7$ & & SUCCESS & FAIL \\
\hline Prediction & SUCCESS & TP $=82$ & FP $=25$ \\
Class & FAIL & FN $=5$ & TN $=7$ \\
\hline
\end{tabular}


Holdout cross-validation may waste datasets and produce a high error rate. Since the aim is generalizing proposed model well without overfitting, therefore 10 -fold cross-validation is used to ensure all observations are used for both training and testing. Each observation is used for testing exactly once.

At the point when the tree is built based on specific features and gives better exactness then the tree can be utilized for feature selection and can consider these features as the best parameters with high predictive power. The best parameters can be determined from datasets using the proposed DDT with the highest accuracy. The perfect accuracies of Iraqi, Por and Math are achieved at iterations 10, 6 and 8, respectively. Table 5 shows 10 iterations and the overall accuracy using 10-fold cross-validation and holdout of the proposed DDT for three datasets.

Table 5. DDT Holdout and 10-Fold Cross-Validation

\begin{tabular}{lllllllllllll}
\hline DDT & Holdout & 1 & 2 & 3 & 4 & 5 & 6 & 7 & 8 & 9 & 10 & 10 Fold AVG \\
\hline Iraq & 88.88 & 58.3 & 58.3 & 91.6 & 83.3 & 91.6 & 91.6 & 91.6 & 91.6 & 83 & 91.6 & 83.3 \\
Por & 61.5 & 92 & 87.5 & 70.3 & 87.5 & 84.3 & 92 & 76.5 & 73.4 & 48.4 & 57.8 & 77 \\
Math & 74.7 & 69 & 71.9 & 61 & 58 & 61.5 & 64 & 64 & 87 & 69 & 66.6 & 67.2 \\
\hline
\end{tabular}

Table 6 shows ID3 based on Holdout and 10- Fold Cross-Validation, from Tables 5 and 6, it can be inferred that the proposed DDT has a higher prediction accuracy than ID3 on the basis of holdout and average of 10-fold cross-validation for two reasons, the first DDT can select the feature based on its importance (weight) taking into account the target class, as opposed to traditional ID3, which chooses a feature of a high category that may not have a predictive classification power, secondly, when there is no combination between features (i.e. sample(value) is empty), the DDT depends on D(ij) for the current value to determine class of leaf nodes, while traditional ID3 decides on a leaf node based on the majority of the class of target attribute, ignoring the tendency of a current value towards a specific class.

Table 6. ID3 Holdout and 10-Fold Cross-Validation

\begin{tabular}{cclllllllllll}
\hline ID3 & Holdout & 1 & 2 & 3 & 4 & 5 & 6 & 7 & 8 & 9 & 10 & 10 Fold AVG \\
\hline Iraq & 83 & 59.3 & 78 & 59.3 & 77 & 86 & 91.6 & 91.6 & 90 & 66.6 & 91.6 & 79 \\
Por & 67 & 87 & 82.8 & 64 & 84 & 81 & 87.5 & 73.4 & 71.8 & 57.8 & 60.9 & 75 \\
Math & 62 & 53.8 & 64 & 74 & 51 & 58.9 & 61.5 & 64 & 66.66 & 69 & 58.9 & 62 \\
\hline
\end{tabular}

In terms of running time, the proposed DDT surpass the traditional ID3, which has faster decision tree construction time than that of ID3. Figure 3, showing that the proposed DDT reduces the time complexity of the traditional ID3 for three datasets since the proposed DDT utilizes simple mathematical expressions incorporates subtraction, addition, and division. All these operations are less computational complexity than ascertaining entropy information that implies calculation of the logarithm algorithm in traditional ID3, which makes DDT useful for improving real-time capability such as online learning systems.

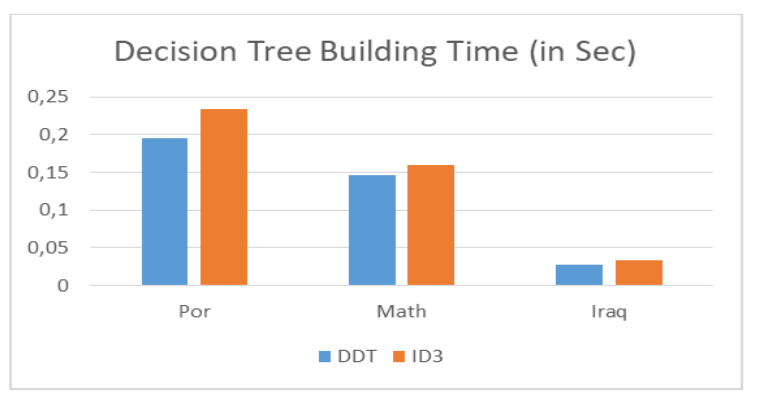

Figure 3. Decision Tree Construction Time for ID3 and DDT

Since the proposed DDT building algorithm selects features locally based on their weight (decisive value), and with relation to the feature selected in earlier stages, so that the features that occur in the DDT are complementary. Therefore, DDT gives a set of extremely important features that lead to a significant increase in the model's predictive accuracy. Table 7 shows the best DDT feature subset, which results in higher 
accuracy for three datasets. Once the best parameter combination has been discovered, a set of classification rules can be extracted from the proposed DDT. These rules help to classify students and foresee the final status of the students.

Table 7. DDT Best Feature Subset

\begin{tabular}{cccl}
\hline Datasets & Accuracy & \#Iteration & \multicolumn{1}{c}{ Features } \\
\hline Iraq & 91.6 & 10 & $\begin{array}{l}\text { Higher Education Willing, sleep Hour, Father Alive, Attendance, Failure Year, Study Hour, Internet } \\
\text { Usage, Parent Meeting, Worry Effect, Arrival Time, Holiday Effect, Transport. }\end{array}$ \\
Por & 92 & 1 & $\begin{array}{l}\text { Fedu, higher, Fjob, absence, study time, health, famrel, walc, dalc, activities, free time, famsize. } \\
\text { gaurdian } \\
\text { Internet, freetime, famrel, failure, health, absence, walc, dalc, study time, romance, reason, health, } \\
\text { medu, higher,paid, schoolsup, gout. }\end{array}$ \\
\hline
\end{tabular}

Table 8 shows a comparison of the proposed DDT with the research work of [24]. This research uses Por dataset from UCI to predict student performance based on eight features G2, G1, failures, higher, Medu, school, studytime, Fedu. In addition, a comparison of the proposed DDT with the research work of [25]. This research uses Math dataset from UCI to predict student performance based on 19 features including the class attribute: sex, famsize, address, Pstatus, Medu, Fedu, Mjob, Fjob, traveltime, studytime, schoolsup, higher, internet, romantic, freetime, Dalc, Walc, health, success. It is clear that the proposed DDT surpass all methods utilized in these researches for two UCI (Por and Math) datasets.

Table 8. Accuracy Comparison of Our Proposed DDT and other Methods for UCI Datasets

\begin{tabular}{cccc}
\hline Dataset & Research Work & Method & Accuracy \\
\hline Por & {$[24](2019)$} & Naïve Bayes & $73.18 \%$ \\
& & Decision Tree & $76.27 \%$ \\
& & RandomTree & $67.95 \%$ \\
& REPTree & $76.73 \%$ \\
& JRip & $74.11 \%$ \\
& & OneR & $76.73 \%$ \\
& & SimpleLogistic & $73.65 \%$ \\
\multirow{4}{*}{ Math } & ZeroR & $30.97 \%$ \\
& Our Proposed Model & The Proposed DDT & $92 \%$ \\
& [25] (2016) & PCF with k- & $65.82 \%$ \\
& \multicolumn{3}{c}{ medoids algorithm } \\
& \multicolumn{3}{c}{ PCF with k-means } \\
& algorithm \\
& Our Proposed Model & The Proposed DDT & $63.50 \%$ \\
& \multicolumn{3}{c}{$87 \%$} \\
\hline
\end{tabular}

\section{CONCLUSION}

This paper proposed an improved ID3 algorithm, which employs attribute weight between attributes and class labels for selection splitting attribute. Constructing the proposed DDT based on feature decisive value ensures that each time important rather than more attribute value is selected. This has a major impact on enhancing classification accuracy. It also has a faster constructing time than classical ID3 which implies time complexity of logarithm computation, as the proposed DDT depends only on calculation attribute frequency of occurrences, which overcomes the limitations of the ID3 algorithm. The proposed algorithm was tested over three datasets. These include Iraqi and two UCI datasets. The obtained results showed that the developed ID3 algorithm beat the traditional ID3 in terms of accuracy and consumed execution time.

\section{REFERENCES}

[1] M. Abdullahi, et al., "The Patterns of Accessing Learning Management System Among Students", Indonesian Journal of Electrical Engineering and Computer Science (IJEECS), Vol. 13, No. 1, ISSN: 2502-4752, DOI: 10.11591/ijeecs. v13.i1. pp15-21, 2019.

[2] M. Gandhi and S.N. Singh, "Predictions in Heart Disease Using Techniques of Data Mining". In Proceedings of the International Conference on Futuristic Trends on Computational Analysis and Knowledge Management, pp. 520-525, 2015.

[3] Özdemir, U. Yavuz, F. Abdulhafidh Dael, "Performance Evaluation of Different Classification Techniques Using Different Datasets", International Journal of Electrical and Computer Engineering (IJECE), Vol. 9, No. 5, ISSN: 2088-8708, DOI: 10.11591/ijece. v9i5. pp. 3584-3590, 2019. 
[4] V.N. Phu, V.T.N. Tran, V.T.N. Chau, N.D. Dat, K.L.D. Duy, “A Decision Tree Using ID3 Algorithm for English Semantic Analysis”. Int. J. Speech Technol., 20, pp. 593-613, 2017.

[5] E. Budiman, Haviluddin, N. Dengan, A. H. Kridalaksana, M. Wati, Purnawansyah, "Performance of Decision Tree C4.5 Algorithm in Student Academic Evaluation”, Springer Nature Singapore Pte Ltd, pp. 380-389, 2018.

[6] T. Roya, S. Sundaramb, P. Ranjana, R. Balasubramaniama, "Prediction of Material Removal in Single Spark Micro-EDM Using Multiple Linear Regression and CART", the 10th International Conference of Precision, Meso, Micro, Nano Engineering, ISBN: 978-93-80689-28-9, 2017.

[7] S. T. Ahmed, R. S. Al-hamdani, M. S. Croock, "Studying of Educational Data Mining Techniques", International Journal of Advanced Research in Science, Engineering and Technology, Vol. 5, Issue 5, 2018.

[8] J. Liang, Z. Shi, "The Information Entropy, Rough Entropy and Knowledge Granulation in Rough Set Theory", Int. J. Uncertain. Fuzziness Knowl.-Based Syst., 12, pp. 37-46, 2008.

[9] T.P. Exarchos, M.G. Tsipouras, C.P. Exarchos, C. Papaloukas, D.I. Fotiadis, L.K. Michalis, "A Methodology for The Automated Creation of Fuzzy Expert Systems for Ischaemic and Arrhythmic Beat Classification Based On a Set of Rules Obtained by A Decision Tree", Artif. Intell. Med., 40, pp.187-200, 2007.

[10] J. Sneyers, T. Schrijvers, B. Demoen, "The computational power and complexity of Constraint Handling Rules", In Proceedings of the 2nd Workshop on Constraint Handling Rules, pp. 3-17, 2005.

[11] U.M. Fayyad, K.B. Irani, "The Attribute Selection Problem in Decision Tree Generation", In Proceedings of the National Conference on Artificial Intelligence, San Jose, CA, USA, pp. 104-110, 1992.

[12] M. Bramer, "Avoiding Overfitting of Decision Trees. In: Principles of Data Mining", book, Springer, DOI https://doi.org/10.1007/978-1-84628-766-4_8, ISBN 978-1-84628-766-4, PP. 119-134, 2007.

[13] L. Yi-bin, W. Ying-ying and R. Xue-wen, "Improvement of ID3 algorithm based on simplified information entropy and coordination degree", Chinese Automation Congress (CAC), Jinan, doi: 10.1109/CAC.2017.8243009, pp. 1526-1530, 2017.

[14] X. Liang, F. Qu, Y. Yang, H. Cai, "An Improved ID3 Decision Tree Algorithm Based on Attribute Weighted", International Conference on Civil, Materials and Environmental Sciences, DOI: 10.2991/cmes-15.2015.167, pp. 613-615, 2015.

[15] P. G. Ahire, S. Kolhe, K. Kirange, H. Karale, A. Bhole, "Implementation of Improved ID3 Algorithm to Obtain More Optimal Decision Tree”, International Journal of Engineering Research and Development, Vol. 11, Issue 02, PP.44-47, 2015.

[16] S. Taha, "Iraqi Student Performance Prediction", Mendeley Data, v1, http://dx.doi.org/10.17632/smgx6s5pwr.1, DOI: 10.17632/smgx6s5pwr.1, 2018.

[17] Saja Taha Ahmed, Rafah Shihab al-Hamdani, Muayad Sadik Croock, "EDM Preprocessing and Hybrid Feature Selection for Improving Classification Accuracy", Journal of Theoretical and Applied Information Technology, Vol.96. No 1 ISSN: 1992-8645, 2019.

[18] P. Cortez and A. Silva.,"Using Data Mining to Predict Secondary School Student Performance", The 5th Future Business Technology Conference (FUBUTEC 2008), pp. 5-12, Porto, 2008.

[19] N. G. M. Jameel, L. E. George, "Detection Phishing Emails Using Features Decisive Values", International Journal of Advanced Research in Computer Science and Software Engineering, Vol.3, Issue.7, 2013.

[20] A. Abdullah, L. E. George, and Imad J. Mohammed," Email Phishing Detection System Using Neural Network", Research Journal of Information Technology, Vol.6, Issue.3, pp. 39-43, 2015.

[21] R. Kohavi, "A study of cross-validation and bootstrap for accuracy estimation and model selection", In Proceedings of International Joint Conference on AI (IJCA), Vol. 14, No. 2, P. 1137-1145, 1995.

[22] S. Raschka, "Model Evaluation, Model Selection, and Algorithm Selection in Machine Learning", arXiv preprint, arXiv:1811.12808v2, 2018.

[23] M. Z. H. Jesmeen, A. Hossen, J. Hossen, J. E. Raja, "AUTO-CDD: Automatic Cleaning Dirty Data Using Machine Learning Techniques", TELKOMNIKA (Telecommunication, Computing, Electronics and Control), Vol.17, No.4, ISSN: 1693-6930, DOI: 10.12928/TELKOMNIKA.v17i4.12780, pp.2076-2086, 2019.

[24] Y. K. Salal, S. M. Abdullaev, M. Kumar, "Educational Data Mining: Student Performance Prediction in Academic", Blue Eyes Intelligence Engineering \& Sciences Publication, International Journal of Engineering and Advanced Technology (IJEAT), ISSN: 2249-8958, Vol.8, Issue-4C, 2019.

[25] N. U. Sati, "Prediction of Students' Success in Mathematics by A Classification Technique Via Polyhedral Conic Functions", International Conference on Research in Education and Science, the Eurasia Proceedings of Educational \& Social Sciences (EPESS), ISSN: 2587-1730, Vol. 5, pp. 190-195, 2016. 